Pacific

Journal of

Mathematics

\title{
A GEOMETRIC MODEL OF AN ARBITRARY REAL CLOSED FIELD
}

\author{
STANISŁAW SPODZIEJA
}




\title{
A GEOMETRIC MODEL OF AN ARBITRARY REAL CLOSED FIELD
}

\author{
STANISŁAW SPODZIEJA
}

\begin{abstract}
We give an elementary construction of any real closed field in terms of Nash function fields. We also give a characterization of any Archimedean field in terms of fields of Nash functions.
\end{abstract}

\section{Introduction}

In the study of Hilbert's 17th problem, orderings of a real field $k$ are of importance (see [Alonso 1986; Alonso et al. 1984; Artin 1927; Artin and Schreier 1927a; 1927b; Bochnak and Efroymson 1980; Bröker 1982; Dubois 1981; Guangxing 2005; Marshall 2003; Prestel and Delzell 2001; Schwartz 1980]). By the Artin-Schreier theorem [Artin 1927; Artin and Schreier 1927a; 1927b], the study of such orderings amounts to considering real closures of $k$. The aim of this article is to construct a universal model of an arbitrary real closed field. To this end, we construct, in terms of Nash functions, all real closures of the rational function fields $k=\mathbb{Q}\left(\Lambda_{T}\right)$, where $\Lambda_{T}=\left(\Lambda_{t}: t \in T\right)$ and $T \neq \varnothing$ is a system of any number of variables. This suffices to achieve our purpose, because any real closed field $R$ is order-preserving isomorphic to a real closure of some field $\mathbb{Q}\left(\Lambda_{T}\right)$ (Corollary 5.5). If $T=\varnothing$, then $\mathbb{Q}\left(\Lambda_{T}\right)=\mathbb{Q}$, and the above is obvious. We assume the Kuratowski-Zorn lemma, so the set $T$ can be well-ordered, provided $T \neq \varnothing$.

L. Bröker [1982] proved in his ultrafilter theorem that there exists a one-to-one correspondence between the family of ultrafilters and the family of orderings in $\mathbb{Q}\left(\Lambda_{T}\right)$, or equivalently with the real closures of $\mathbb{Q}\left(\Lambda_{T}\right)$. We prove that there exists a one-to-one correspondence between the family of orderings in $\mathbb{Q}\left(\Lambda_{T}\right)$ and the family of plain filters (Theorem 5.2, Proposition 2.4, and Corollary 2.5). By a plain filter we mean a filter $\Omega$ of subsets of $\mathbb{R}^{T}$ with these properties:

(1) Any $U \in \Omega$ is a nonempty open connected semialgebraic set.

(2) For any algebraic set $V \subsetneq \mathbb{R}^{T}$, where $V=P^{-1}(0)$ and $P \in \mathbb{Q}\left[\Lambda_{T}\right]$, some connected component of $\mathbb{R}^{T} \backslash V$ belongs to $\Omega$.

This research was partially supported by the program POLONIUM 2009-2010.

MSC2000: primary 14P10, 14P20; secondary 32C07.

Keywords: Nash function, semialgebraic set, real closed field, ordering. 
(3) For any $U_{1}, U_{2} \in \Omega$, there exists $U_{3} \in \Omega$ such that $U_{3} \subset U_{1} \cap U_{2}$.

The correspondence between orderings and plain filters is as follows: For any ordering $\succ$ of $\mathbb{Q}\left(\Lambda_{T}\right)$, there exists a unique plain filter $\Omega$ such that $f \succ 0$ if and only if $f>0$ on some $U \in \Omega$, where $>$ is the usual ordering on $\mathbb{R}$. Conversely, any plain filter $\Omega$ determines a unique ordering $\succ$ of $\mathbb{Q}\left(\Lambda_{T}\right)$ in this way.

The main result of this article is Theorem 5.2, where we give a construction of any real closure of $\mathbb{Q}\left(\Lambda_{T}\right)$ in terms of Nash functions. The main idea and motivation for the above considerations was a geometric construction of the algebraic closure of $\mathbb{C}\left(\Lambda_{1}, \ldots, \Lambda_{m}\right)$ [Spodzieja 1996]. More precisely, for any plain filter $\Omega$ of open connected semialgebraic sets and any $U \in \Omega$, the ring $\mathcal{N}(U)$ of $\mathbb{Q}$-Nash functions (see Section 1) on $U$ is a domain. In $\bigcup_{U \in \Omega} \mathcal{N}(U)$, we introduce an equivalence relation $\sim:\left(f_{1}: U_{1} \rightarrow \mathbb{R}\right) \sim\left(f_{2}: U_{2} \rightarrow \mathbb{R}\right)$ if and only if $\left.f_{1}\right|_{U_{3}}=\left.f_{2}\right|_{U_{3}}$ for some $U_{3} \in \Omega$. The set $\mathcal{N}_{\Omega}$ of equivalence classes of $\sim$ with the usual operations of addition and multiplication is a field, which is a real closure of $\mathbb{Q}\left(\Lambda_{T}\right)$ (see Theorem 5.2, and compare [Spodzieja 1996, Theorem 2.4 and Corollary 2.5]). One can view $\mathcal{N}_{\Omega}$ as the inverse limit of the étale topology $\bigcup_{U \in \Omega} \mathcal{N}(U)$ of $\mathbb{R}^{T}$ [Grothendieck 1967].

In Section 3, we prove that an ordering $\succ$ of $\mathbb{Q}\left(\Lambda_{T}\right)$ is Archimedean if and only if the set $\bigcap_{U \in \Omega} U$ is nonempty for the plain filter $\Omega$ determining $\succ$; and if that is the case, this set has exactly one point (Theorem 3.1). In Section 4, we give some examples of non-Archimedean orderings corresponding to the one in [Spodzieja 1996].

\section{Preliminaries}

Let $\mathbb{K}$ be the field $\mathbb{Q}$ of rational, $\mathbb{R}$ of real, or $\mathbb{C}$ of complex numbers. Let $T$ be a nonempty set. We denote by $\Lambda_{T}=\left(\Lambda_{t}: t \in T\right)$ a system of independent variables $\Lambda_{t}$, by $\mathbb{K}\left[\Lambda_{T}\right]$ the ring of polynomials in $\Lambda_{T}$ over $\mathbb{K}$, and by $\mathbb{K}\left(\Lambda_{T}\right)$ the quotient field of $\mathbb{K}\left[\Lambda_{T}\right]$. Note that for any $P \in \mathbb{K}\left(\Lambda_{T}\right)$, we have $P \in \mathbb{K}\left(\Lambda_{t_{1}}, \ldots, \Lambda_{t_{m}}\right)$ for some finite number of indices $t_{1}, \ldots, t_{m} \in T$.

We denote by $\mathbb{K}^{T}$ the set of all functions $T \rightarrow \mathbb{K}$ equipped with the unique topology for which all projections $\mathbb{K}^{T} \ni x \mapsto x(t) \in \mathbb{K}, t \in T$ are continuous.

Let $\mathbb{L}$ be a subfield of $\mathbb{K}$. A subset of $\mathbb{K}^{T}$ is called $\mathbb{L}$-algebraic, or simply algebraic if $\mathbb{L}=\mathbb{K}$, when it is defined by a finite system of equations $P=0$, where $P \in \mathbb{L}\left[\Lambda_{T}\right]$. Any $\mathbb{L}$-algebraic set in $\mathbb{K}^{T}$ is of the form $\left\{x \in \mathbb{K}^{T}:\left(x\left(t_{1}\right), \ldots, x\left(t_{m}\right)\right) \in V\right\}$, where $m \in \mathbb{N}, t_{1}, \ldots, t_{m} \in T$, and $V \subset \mathbb{K}^{m}$ is an $\mathbb{L}$-algebraic subset of $\mathbb{K}^{m}$.

If $\mathbb{L}$ is a subfield of $\mathbb{R}$, then we assume that $\mathbb{L}$ is an ordered field with order induced from $\mathbb{R}$.

Let $\mathbb{L}$ be a subfield of $\mathbb{R}$. A subset of $\mathbb{R}^{T}$ is called $\mathbb{L}$-semialgebraic when it is defined by a finite alternative of finite systems of inequalities $P>0$ or $P \geq 0$, where $P \in \mathbb{L}\left[\Lambda_{T}\right]$. Analogously to the above, any $\mathbb{L}$-semialgebraic set in $\mathbb{R}^{T}$ is of the form 
$\left\{x \in \mathbb{R}^{T}:\left(x\left(t_{1}\right), \ldots, x\left(t_{m}\right)\right) \in X\right\}$, where $m \in \mathbb{N}, t_{1}, \ldots, t_{m} \in T$, and $X \subset \mathbb{R}^{m}$ is an $\mathbb{L}$-semialgebraic subset of $\mathbb{R}^{m}$. A set is called open basic $\mathbb{L}$-semialgebraic if it has the form $\left\{x \in \mathbb{R}^{T}: g_{i}(x)>0, i=1, \ldots, n\right\}$, for some $n \in \mathbb{N}$ and $g_{i} \in \mathbb{L}\left[\Lambda_{T}\right]$, $i=1, \ldots, n$.

We now list some basic properties of algebraic and semialgebraic sets in infinitedimensional real vector spaces, which follow easily from their analogues in finitedimensional spaces [Benedetti and Risler 1990; Bochnak et al. 1987; Bochnak and Efroymson 1980; Efroymson 1974; 1976; 1981; Mostowski 1976; Prestel and Delzell 2001; Tancredi and Tognoli 2006; Tworzewski 1990].

Proposition 1.1. Let $\mathbb{L}$ be a subfield of $\mathbb{R}($ or $\mathbb{K}$ in (a)).

(a) The family of $\mathbb{L}$-algebraic sets in $\mathbb{K}^{T}$ is closed with respect to union and intersection of a finite number of sets.

(b) The family of $\mathbb{L}$-semialgebraic sets in $\mathbb{R}^{T}$ is closed with respect to complement, union, and intersection of a finite number of sets.

(c) (Tarski-Seidenberg) Let $\pi_{t_{1}, \ldots, t_{m}}: \mathbb{R}^{T} \ni x \mapsto\left(x\left(t_{1}\right), \ldots, x\left(t_{m}\right)\right) \in \mathbb{R}^{m}$, where $t_{1}, \ldots, t_{m} \in T$. If $X \subset \mathbb{R}^{T}, Y \subset \mathbb{R}^{m}$ are $\mathbb{L}$-semialgebraic sets, then $\pi_{t_{1}, \ldots, t_{m}}(X)$ and $\pi_{t_{1}, \ldots, t_{m}}^{-1}(Y)$ are $\mathbb{L}$-semialgebraic sets, too.

(d) For any $\mathbb{L}$-semialgebraic set $X \subset \mathbb{R}^{T}$, the interior Int $X$, closure $\bar{X}$, and the boundary $\partial X$ are $\mathbb{L}$-semialgebraic sets.

Let $\mathbb{L}$ be a subfield of $\mathbb{R}$. A function $f: U \rightarrow \mathbb{R}$, where $U \subset \mathbb{R}^{T}$ is an open $\mathbb{L}$-semialgebraic set, is called an $\mathbb{L}$-Nash function if $f$ is analytic and there exists a nonzero polynomial $P \in \mathbb{L}\left[\Lambda_{T}, Z\right]$ such that $P(\lambda, f(\lambda))=0$ for $\lambda \in U$. In fact, $f$ depends on a finite number of variables, so the analyticity of $f$ is clear. The ring of ¿-Nash functions in $U$ is denoted by $\mathcal{N}^{\mathbb{L}}(U)$.

The next result follows via R. Thom's lemma (see for instance [Bochnak et al. 1987, Proposition 2.5.4 and the arguments of Theorems 2.3.6 and 2.4.4]) from the fact that any $\mathbb{L}$-semialgebraic set in a finite-dimensional vector space over $\mathbb{R}$ is the disjoint union of a finite number of $\mathbb{L}$-semialgebraic sets which are homeomorphic to Cartesian products of intervals.

Proposition 1.2. Let $\mathbb{Q}$ be a subfield of $\mathbb{R}$. Any connected component of an $\mathbb{L}-$ semialgebraic subset of $\mathbb{R}^{T}$ is $\mathbb{L}$-semialgebraic.

A function $f: U \rightarrow \mathbb{C}$, where $U \subset \mathbb{C}^{T}$ is an open set, is called a $\mathbb{C}$-Nash function if $f$ is holomorphic and there exists a nonzero polynomial $P \in \mathbb{C}\left[\Lambda_{T}, Z\right]$ such that $P(\lambda, f(\lambda))=0$ for $\lambda \in U$. The ring of $\mathbb{C}$-Nash functions in $U$ is denoted by $\mathcal{N}^{\mathbb{C}}(U)$.

For the basic properties of Nash functions and semialgebraic sets in finitedimensional vector spaces, see, for instance, [Benedetti and Risler 1990; Bochnak et al. 1987; Bochnak and Efroymson 1980; Efroymson 1974; 1976; 1981; Mostowski 
1976; Nash 1952; Tancredi and Tognoli 2006; Tworzewski 1990]. From these properties, we immediately obtain:

Proposition 1.3. Let $\mathbb{K}=\mathbb{R}$ or $\mathbb{K}=\mathbb{C}$, let $\mathbb{L}$ be a subfield of $\mathbb{K}$, and let $U \subset \mathbb{K}^{T}$ be an open connected set. Then $\mathcal{N}^{\mathbb{K}}(U)$ is a domain, provided $U$ is semialgebraic when $\mathbb{K}=\mathbb{R}$. In particular $\mathcal{N}^{\mathbb{Q}}(U)$ is a domain.

\section{Orderings in $\mathbb{Q}\left(\Lambda_{T}\right)$}

Let $T$ be a nonempty set. A family $\Omega$ of subsets of $\mathbb{R}^{T}$ will be called a $c$-filter (connected sets filter) if it satisfies these conditions:

(i) Any $U \in \Omega$ is a nonempty open connected $\mathbb{Q}$-semialgebraic set.

(ii) For any $\mathbb{Q}$-algebraic set $V \varsubsetneqq \mathbb{R}^{T}$, there exists $U \in \Omega$ such that $V \cap U=\varnothing$.

(iii) For any $U_{1}, U_{2} \in \Omega$, there exists $U_{3} \in \Omega$ such that $U_{3} \subset U_{1} \cap U_{2}$.

Proposition 2.1. Let $\Omega$ be a c-filter of subsets of $\mathbb{R}^{T}$. The set $\partial \Omega:=\bigcap_{U \in \Omega} \bar{U}$ has at most one point. Moreover, whenever $T$ is a finite set, $\partial \Omega \neq \varnothing$ if and only if there exists a bounded set $U \in \Omega$.

Proof. If $x_{1}, x_{2} \in \partial \Omega$ with $x_{1} \neq x_{2}$, then for some polynomial $f \in \mathbb{Q}\left[\Lambda_{T}\right]$, we have $f\left(x_{1}\right)<0<f\left(x_{2}\right)$. Hence, for some $W \in \Omega$ such that $W \cap f^{-1}(0)=\varnothing$, we have both $f(x)<0$ and $f(x)>0$ for some $x \in W$. This contradiction gives the first part of the assertion.

Now let $T=\left\{t_{1}, \ldots, t_{m}\right\}$. Suppose that $\partial \Omega \neq \varnothing$ and each $W \in \Omega$ is an unbounded set. Take $x_{0} \in \partial \Omega$, and let $f=\left(\Lambda_{T}\right)=\Lambda_{t_{1}}^{2}+\cdots+\Lambda_{t_{m}}^{2}-r$, where $r \in \mathbb{Q}$ and $r>x_{0}^{2}\left(t_{1}\right)+\cdots+x_{0}^{2}\left(t_{m}\right)$. Then $f^{-1}(0) \cap W=\varnothing$ for some $W \in \Omega$. Since $W$ is a connected unbounded set, $x_{0}$ is not an accumulation point of $W$. This contradicts the choice of $x_{0}$. Now assume that some $W \in \Omega$ is bounded. Then it is easy to see that there exists a sequence of nonempty compact sets $C_{1} \supset C_{2} \supset \cdots$ with diameters decreasing to 0 and such that $U \cap C_{n} \neq \varnothing$ for all $U \in \Omega$ and $n \in \mathbb{N}$. Then there exists $x \in \bigcap_{n \in \mathbb{N}} C_{n}$ belonging to $\partial \Omega$.

Let us fix a c-filter $\Omega$ and define a relation $\succ_{\Omega}$ in $\mathbb{Q}\left(\Lambda_{T}\right)$ by

$$
\begin{aligned}
& f \succ_{\Omega} 0 \Longleftrightarrow \text { there exists } U \in \Omega \text { such that } f(x)>0 \text { for all } x \in U, \\
& f \succ_{\Omega} g \Longleftrightarrow f-g \succ_{\Omega} 0 .
\end{aligned}
$$

Let $\Omega$ be a family of subsets of $\mathbb{R}^{T}$. If an ordering $\succ$ of $\mathbb{Q}\left(\Lambda_{T}\right)$ satisfies $f \succ 0$ if and only if $f>0$ on some $U \in \Omega$, we say that $\Omega$ determines the ordering $\succ$.

Lemma 2.2. The relation $\succ_{\Omega}$ is an ordering in $\mathbb{Q}\left(\Lambda_{T}\right)$, or in other words, a total ordering satisfying

$$
f \succ_{\Omega} g \Rightarrow f+h \succ_{\Omega} g+h \text { and } f \succ_{\Omega} 0, g \succ_{\Omega} 0 \Rightarrow f g \succ_{\Omega} 0 .
$$


Proof. The relation $\succ_{\Omega}$ is well-defined. Indeed, if $f \in \mathbb{Q}\left(\Lambda_{T}\right)$ and $f \neq 0$, then the union of the sets of zeros and poles of $f$ is contained in some $\mathbb{Q}$-algebraic set $V \varsubsetneqq \mathbb{R}^{m}$. Hence, by (i) and (ii), for some $U \in \Omega$, the values $f(x)$ have a fixed sign for all $x \in U$. Moreover, if for some $U_{1}, U_{2} \in \Omega$ we have $f(x)>0$ for $x \in U_{1}$ and $f(x) \leq 0$ for $x \in U_{2}$, then $0<f(x) \leq 0$ for $x \in U_{1} \cap U_{2}$, and $U_{1} \cap U_{2} \neq \varnothing$ by (iii). This is impossible. It is easy to see that the remaining conditions are also satisfied.

Proposition 2.3. Let $\Omega_{1}, \Omega_{2}$ be c-filters. If the orderings $\succ_{\Omega_{1}}$ and $\succ_{\Omega_{2}}$ are equal, then $\Omega=\left\{U \cup W: U \in \Omega_{1}, W \in \Omega_{2}\right\}$ is a c-filter determining the ordering $\succ_{\Omega_{1}}$.

Proof. Since $\Omega_{1}$ and $\Omega_{2}$ are c-filters, it suffices to prove that $U \cap W \neq \varnothing$ for all $U \in \Omega_{1}$ and $W \in \Omega_{2}$. Suppose $U \cap W=\varnothing$ for some $U \in \Omega_{1}$ and $W \in \Omega_{2}$. Let $U=U_{1} \cup \cdots \cup U_{k} \cup V$ be a decomposition of $U$ into disjoint basic open semialgebraic sets $U_{1}, \ldots, U_{k}$ and a semialgebraic set $V$ included in an algebraic set. By (i) and (ii), there exists $U^{\prime} \in \Omega_{1}$ such that $U^{\prime} \subset U_{i}$ for some $i \in\{1, \ldots, k\}$. Since $U_{i}=\left\{x \in \mathbb{R}^{T}: f_{j}(x)>0, j=1, \ldots, n\right\}$ for some $f_{1}, \ldots, f_{n} \in \mathbb{Q}\left[\Lambda_{T}\right]$, by the assumption we have $f_{1}, \ldots, f_{n} \succ_{\Omega_{1}} 0$, and so there exists $W_{1} \in \Omega_{2}$ such that $f_{j}(x)>0$ for all $x \in W_{1}$ and $j=1, \ldots, n$. By (iii), there exists $W_{2} \in \Omega_{2}$ such that $W_{2} \subset W \cap W_{1}$ and $f_{j}(x)>0$ for all $j=1, \ldots, n$ and $x \in W_{2}$. Thus $W_{2} \subset U$, which contradicts the assumption.

Now let $\succ$ be an ordering in $\mathbb{Q}\left(\Lambda_{T}\right)$, and let

$u_{\succ}=\left\{\bigcap_{i=1}^{n} f_{i}^{-1}((0,+\infty)) \subset \mathbb{R}^{T}: f_{i} \in \mathbb{Q}\left(\Lambda_{T}\right), f_{i} \succ 0\right.$ for $\left.i=1, \ldots, n, n \in \mathbb{N}\right\}$,

where we regard $f \in \mathbb{Q}\left(\Lambda_{T}\right)$ as a function $f: \mathbb{R}^{T} \rightarrow \mathbb{R}$. By the definition of $\boldsymbol{u}_{\succ}$ and the Tarski transfer principle (see [Tarski 1948; Seidenberg 1954]), we find that $\varnothing \notin u_{\succ}$. Moreover, the relation $\succ$ is defined by

$$
f \succ 0 \Longleftrightarrow \text { there exists } U \in U_{\succ} \text { such that } f(x)>0 \text { for all } x \in U .
$$

The sets of the family $\boldsymbol{U}_{\succ}$ may be disconnected, so $u_{\succ}$ is not a c-filter. We will prove that the ordering $\succ$ is defined by some c-filter.

Proposition 2.4. There exists a unique c-filter $\Omega$ with the following properties:

(a) For any $f \in \mathbb{Q}\left(\Lambda_{T}\right)$, we have $f \succ_{\Omega} 0$ if and only if $f \succ 0$.

(b) For any $U \in \Omega$, there exists a $\mathbb{Q}$-algebraic set $V \subsetneq \mathbb{R}^{T}$ such that $U$ is a connected component of $\mathbb{R}^{T} \backslash V$.

(c) For any $\mathbb{Q}$-algebraic set $V \subsetneq \mathbb{R}^{T}$, some connected component of $\mathbb{R}^{T} \backslash V$ belongs to $\Omega$.

Proof. Let $\mathscr{F}$ be the family of all connected components of sets $U \in U_{\succ}$. 
Claim 1. Every $U \in \mathcal{U}_{\succ}$ has a connected component $U_{0}$ such that $U_{0} \cap W \neq \varnothing$ for any $W \in u_{\succ}$.

Let $U \in \mathcal{U}_{\succ}$ and let $U=U_{1} \cup \cdots \cup U_{n}$ be the decomposition into connected components. Assume to the contrary that there exist $W_{1}, \ldots, W_{n} \in \mathcal{U}_{\succ}$ such that $U_{i} \cap W_{i}=\varnothing$ for $i=1, \ldots, n$. Then $U \cap W_{1} \cap \cdots \cap W_{n}=\varnothing$, which is impossible. This gives Claim 1.

Claim 2. Each $U \in \mathcal{U}_{\succ}$ has exactly one connected component $S_{U}$ that intersects every $W \in u_{\succ}$.

Let $U \in \mathcal{U}_{\succ}$, and let $U_{1}, \ldots, U_{p}$ be the connected components of $U$. Then

$$
U=\bigcap_{l=1}^{s}\left\{x \in \mathbb{R}^{T}: g_{l}(x)>0\right\}
$$

for some nonzero polynomials $g_{l} \in \mathbb{Q}\left[\Lambda_{T}\right]$, with $g_{l} \succ 0$ for $l=1, \ldots, s$, and

$$
U_{i}=\left[f_{i}^{-1}(0) \cap U_{i}\right] \cup \bigcup_{j=1}^{n} \bigcap_{k=1}^{m}\left\{x \in \mathbb{R}^{T}: f_{i, j, k}(x)>0\right\}, \quad i=1, \ldots, p,
$$

for some nonzero polynomials $f_{i}, f_{i, j, k} \in \mathbb{Q}\left[\Lambda_{T}\right]$. Denote by $\epsilon_{i, j, k}$ the sign of $f_{i, j, k}$ in the ordering $\succ$. Then $\epsilon_{i, j, k} \neq 0$ and $\epsilon_{i, j, k} f_{i, j, k} \succ 0$ for any $i, j, k$. Observe that for some $i \in\{1, \ldots, p\}$ and $j \in\{1, \ldots, n\}$, we have $f_{i, j, k} \succ 0$ for $k=1, \ldots, m$. Indeed, in the opposite case,

$$
\varnothing=\bigcap_{l=1}^{s} \bigcap_{i=1}^{p} \bigcap_{j=1}^{n} \bigcap_{k=1}^{m}\left\{x \in \mathbb{R}^{T}: g_{l}(x)>0, \epsilon_{i, j, k} f_{i, j, k}(x)>0\right\} \in \mathcal{U}_{\succ},
$$

which is impossible. So, for some $i_{0} \in\{1, \ldots, p\}$ and $j_{0} \in\{1, \ldots, n\}$,

$$
U^{\prime}=\bigcap_{k=1}^{m}\left\{x \in \mathbb{R}^{T}: f_{i_{0}, j_{0}, k}(x)>0\right\} \in U_{\succ},
$$

and $U^{\prime} \cap U_{j}=\varnothing$ for $j \neq j_{0}$. Hence, by Claim $1, S_{U}=U_{j_{0}}$ is the unique connected component of $U$ satisfying Claim 2.

Claim 3. The family $\Omega=\left\{S_{U}: U \in \mathcal{U}_{\succ}\right\}$ is a c-filter.

Since for every $\mathbb{Q}$-algebraic set $V \subset \mathbb{R}^{T}$ there exists $U \in \mathcal{U}_{\succ}$ such that $U \cap V=\varnothing$, we have $S_{U} \cap V=\varnothing$. Hence, it suffices to prove that for any $S_{U_{1}}, S_{U_{2}} \in \Omega$, there exists $S_{U_{3}} \in \Omega$ contained in $S_{U_{1}} \cap S_{U_{2}}$. Indeed, by the argument of Claim 2, there exist $W_{1}, W_{2} \in U_{\succ}$ such that $W_{1} \subset S_{U_{1}}$ and $W_{2} \subset S_{U_{2}}$. Hence, $S_{W_{1} \cap W_{2}} \subset W_{1} \cap W_{2} \subset$ $S_{U_{1}} \cap S_{U_{2}}$ and $S_{W_{1} \cap W_{2}} \in \Omega$.

Claim 4. The c-filter $\Omega$ defined in Claim 3 satisfies the assertion of Proposition 2.4. 
Part (a) is obvious.

Let $U \in U_{\succ}$ be of the form (1), $f=g_{1} \ldots g_{s}$, and $V=f^{-1}(0)$. Then, by the definition of $S_{U}$, we see that $S_{U}$ is a connected component of $\mathbb{R}^{T} \backslash V$. This gives (b).

Let $V=f^{-1}(0)$ be a $\mathbb{Q}$-algebraic subset of $\mathbb{R}^{T}$. Then $U=\left\{x \in \mathbb{R}^{T}: f^{2}(x)>0\right\}=$ $\mathbb{R}^{T} \backslash V \in U_{\succ}$ and $S_{U} \in \Omega$ is a connected component of $\mathbb{R}^{T} \backslash V$. This gives (c) and completes the proof.

We call the c-filter $\Omega$ defined in Proposition 2.4 the plain filter for the ordering $\succ$ and denote it by $\Omega_{\succ}$.

From Proposition 2.4, we immediately obtain:

Corollary 2.5. The mapping $\succ \mapsto \Omega_{\succ}$ is a one-to-one correspondence between the set of orderings of $\mathbb{Q}\left(\Lambda_{T}\right)$ and the set of plain filters.

Remark 2.6. From the ultrafilter theorem [Bröker 1982], we see that for any ultrafilter $\mathscr{F}$ of subsets of $\mathbb{R}^{T}$, there exists a plain filter $\Omega \subset \mathscr{F}$.

Remark 2.7. It is easy to observe that the statements of this section hold with $\mathbb{Q}$ replaced by $\mathbb{R}$.

\section{Archimedean orderings in $\mathbb{Q}\left(\Lambda_{T}\right)$}

Let $\succ$ be an ordering of $\mathbb{Q}\left(\Lambda_{T}\right)$. Then one can assume that $T$ is linearly ordered by

$$
t_{1} \succ t_{2} \Longleftrightarrow \Lambda_{t_{1}} \succ \Lambda_{t_{2}} .
$$

If $f \succ g$, then we also write $g \prec f$.

Theorem 3.1. The following conditions are equivalent:

(a) The field $\left(\mathbb{Q}\left(\Lambda_{T}\right), \succ\right)$ is Archimedean.

(b) There exists $x_{\succ} \in \partial \Omega_{\succ}$ such that the set of coordinates of $x_{\succ}$ is algebraically independent over $\mathbb{Q}$.

(c) There exists $x_{\succ} \in \partial \Omega_{\succ}$ such that $f \succ 0$ if and only if $f\left(x_{\succ}\right)>0$.

(d) There exists $x_{\succ} \in \partial \Omega_{\succ}$ such that $x_{\succ} \in U$ for any $U \in \Omega_{\succ}$.

Proof. Assume (a). Then for any $t_{1}, \ldots, t_{n} \in T$ with $t_{1} \prec \cdots \prec t_{n}$, and for the projection $\pi_{t_{1}, \ldots, t_{n}}: \mathbb{R}^{T} \mapsto\left(x\left(t_{1}\right), \ldots, x\left(t_{n}\right)\right) \in \mathbb{R}^{n}$, the family

$$
\Omega_{t_{1}, \ldots, t_{n}}=\left\{\pi_{t_{1}, \ldots, t_{n}}(U): U \in \Omega\right\}
$$

determines an Archimedean order in $\mathbb{Q}\left(\Lambda_{t_{1}}, \ldots, \Lambda_{t_{n}}\right)$. Thus for some $W \in \Omega_{t_{1}, \ldots, t_{n}}$, the function $f=\Lambda_{t_{1}}^{2}+\cdots+\Lambda_{t_{n}}^{2}$ is bounded on $W$. So the set $W$ is bounded. Hence, by Proposition 2.1, there exists $\left(x_{1}, \ldots, x_{n}\right) \in \partial \Omega_{t_{1}, \ldots, t_{n}}$. Since the projections $\pi_{t_{1}, \ldots, t_{n}}$ are open, it is easy to observe that, for $t_{k_{1}}, \ldots, t_{k_{j}} \in\left\{t_{1}, \ldots, t_{n}\right\}$ with $t_{k_{1}} \prec \cdots \prec t_{k_{j}}$, we have $\left(x_{k_{1}}, \ldots, x_{k_{j}}\right) \in \partial \Omega_{t_{k_{1}}, \ldots, t_{k_{j}}}$. Consequently, there 
exists $x \in \mathbb{R}^{T}$ such that for any $t_{1}, \ldots, t_{n} \in T$ with $t_{1} \prec \cdots \prec t_{n}$, we have $\pi_{t_{1}, \ldots, t_{n}}(x) \in \partial \Omega_{t_{1}, \ldots, t_{n}}$. Summing up, $x \in \partial \Omega$. The set of coordinates of $x$ is algebraically independent over $\mathbb{Q}$ : otherwise, $f(x)=0$ for some nonzero polynomial $f \in \mathbb{Q}\left[\Lambda_{T}\right]$, and so $f$ is infinitesimal. This contradicts (a) and gives (b).

Assume (b). Then any nonzero $f \in \mathbb{Q}\left(\Lambda_{T}\right)$ with $f \succ 0$ is defined at $x_{\succ}$. Moreover, $f\left(x_{\succ}\right) \neq 0$, so $f\left(x_{\succ}\right)>0$. Conversely, assume that $f\left(x_{\succ}\right)>0$. Then obviously for some connected component $U$ of $f^{-1}(0,+\infty)$, we have $U \in \Omega_{\succ}$ and $f(x)>0$ for $x \in U$. Summing up, we obtain (c).

The implication (c) $\Rightarrow$ (d) is trivial.

Now assume (d). Then we immediately obtain (b), and hence, no $f \in \mathbb{Q}\left(\Lambda_{T}\right)$ is infinitesimal, and the field $\left(\mathbb{Q}\left(\Lambda_{T}\right), \succ\right)$ is Archimedean. This gives (a) and completes the proof.

Remark 3.2. The assertion of Theorem 3.1 also holds for every c-filter determining $\succ$ in place of the plain filter $\Omega_{\succ}$.

Theorem 3.1 implies:

Corollary 3.3. Let $T$ be a finite set. Then the set of Archimedean orderings of $\mathbb{Q}\left(\Lambda_{T}\right)$ is a dense subset of the space of orderings in $\mathbb{Q}\left(\Lambda_{T}\right)$ in the path topology (see, for instance, [Marshall 2008]) of the real spectrum $\operatorname{Sper}\left(\mathbb{Q}\left[\Lambda_{T}\right]\right)$.

\section{Examples of non-Archimedean orderings}

Let $m$ be a fixed positive integer and $\Lambda$ a system of $m$ variables $\Lambda_{1}, \ldots, \Lambda_{m}$.

Take any $P \in \mathbb{R}[\Lambda]$. Let $\Gamma_{P} \subset \mathbb{R}^{m}$ be a set defined by

$$
\Gamma_{P}=\left\{\left(\lambda_{1}, \ldots, \lambda_{m}\right) \in \mathbb{R}^{m}: P\left(\lambda_{1}, \ldots, \lambda_{m-1}, \lambda_{m}+\gamma\right)=0 \text { for some } \gamma \in[0, \infty)\right\} \text {. }
$$

We define a polynomial $\omega(P) \in \mathbb{R}\left[\Lambda_{1}, \ldots, \Lambda_{m-1}\right]$ (or a number $\omega(P) \in \mathbb{R}$ if $m=1$ ) by $\omega(P)=0$ for $P=0$, and $\omega(P)=P_{0}$ for $P \neq 0$, where

$$
P=P_{0} \Lambda_{m}^{d}+P_{1} \Lambda_{m}^{d-1}+\cdots+P_{d}
$$

and $P_{i} \in \mathbb{R}\left[\Lambda_{1}, \ldots, \Lambda_{m-1}\right]$ (or $P_{i} \in \mathbb{R}$ if $m=1$ ) for $i=0, \ldots, d$ and $P_{0} \neq 0$.

Let us define sets $W_{P} \subset \mathbb{R}^{m}$, for $P \in \mathbb{R}[\Lambda]$. The definition will be inductive with respect to the number of variables $\Lambda_{1}, \ldots, \Lambda_{m}$. For $P \in \mathbb{R}[\Lambda]$, we put

$$
W_{P}= \begin{cases}\mathbb{R} \backslash \Gamma_{P} \subset \mathbb{R} & \text { if } m=1, \\ \left(\mathbb{R}^{m} \backslash \Gamma_{P}\right) \cap\left(W_{\omega(P)} \times \mathbb{R}\right) \subset \mathbb{R}^{m} & \text { if } m>1 .\end{cases}
$$

By the Tarski-Seidenberg theorem - see Proposition 1.1(c) - the sets $W_{P}$ are semialgebraic for all $P \in \mathbb{R}[\Lambda]$.

Analogously to Theorem 1.1 of [Spodzieja 1996], we prove the following proposition, which gives an example of c-filter. 
Proposition 4.1. The family $\mathscr{W}=\left\{W_{P}: P \in \mathbb{R}[\Lambda]\right\}$ satisfies these conditions:

$R_{0} . W_{P} \subset\left\{\lambda \in \mathbb{R}^{m}: P(\lambda) \neq 0\right\}$.

$R_{1} . W_{P} \cap W_{Q}=W_{P Q}$.

$R_{2}$. For $P \neq 0, W_{P}$ is an unbounded subset of $\mathbb{R}^{m}$.

$R_{3}$. For $P \neq 0, W_{P}$ is an open, connected and simply connected set.

Moreover, one can demand that

$R_{4} . W_{P}=\mathbb{R}^{m}$ for $P=$ const, $P \neq 0$.

In particular, $\mathcal{W}$ contains the $c$-filter

$$
\Omega=\left\{W_{P}: P \in \mathbb{Q}[\Lambda]\right\}
$$

Lemma 4.2. Let $1 \leq i_{1}<\cdots<i_{m} \leq n$, and let $P \in \mathbb{R}\left[\Lambda_{i_{1}}, \ldots, \Lambda_{i_{m}}\right]$. Let $Q \in \mathbb{R}\left[\Lambda_{1}, \ldots, \Lambda_{n}\right]$ be a polynomial of the form

$$
Q\left(x_{1}, \ldots, x_{n}\right)=P\left(x_{i_{1}}, \ldots, x_{i_{m}}\right), \quad\left(x_{1}, \ldots, x_{n}\right) \in \mathbb{R}^{n} .
$$

Then $W_{P} \subset \mathbb{R}^{m}, W_{Q} \subset \mathbb{R}^{n}$, and

$$
W_{Q} \subset\left\{\left(x_{1}, \ldots, x_{n}\right) \in \mathbb{R}^{n}:\left(x_{i_{1}}, \ldots, x_{i_{m}}\right) \in W_{P}\right\} .
$$

Proof. For $P=0$ or $n=m$, the assertion is trivial. Assume that $P \neq 0$ and $n>m$. Consider the case $n=m+1$. Then there exists $1 \leq j \leq n$ such that

$$
\left(\Lambda_{i_{1}}, \ldots, \Lambda_{i_{m}}\right)=\left(\Lambda_{1}, \ldots, \Lambda_{n-j}, \Lambda_{n-j+2}, \ldots, \Lambda_{n}\right)
$$

under the obvious convention for $j=1$ and $j=n$. Denote the $i$-th iteration of $\omega$ by $\omega^{i}$, where $\omega^{0}(P)=P$. Then, for $\left(x_{1}, \ldots, x_{n-i}\right) \in \mathbb{R}^{n-i}$,

$\omega^{i}(Q)\left(x_{1}, \ldots, x_{n-i}\right)= \begin{cases}\omega^{i}(P)\left(x_{1}, \ldots, x_{n-j}, x_{n-j+2}, \ldots, x_{n-i}\right) & \text { if } 0 \leq i \leq j-2, \\ \omega^{i}(P)\left(x_{1}, \ldots, x_{n-j}\right) & \text { if } i=j-1, \\ \omega^{i-1}(P)\left(x_{1}, \ldots, x_{n-i}\right) & \text { if } j \leq i \leq n .\end{cases}$

Hence,

$$
\Gamma_{\omega^{i}(Q)}=\left\{\left(x_{1}, \ldots, x_{n-i}\right) \in \mathbb{R}^{n-i}:\left(x_{1}, \ldots, x_{n-j}, x_{n-j+2}, \ldots, x_{n-i}\right) \in \Gamma_{\omega^{i}(P)}\right\}
$$

for $0 \leq i \leq j-2$, and

$$
\Gamma_{\omega^{j-1}(Q)}=\left\{\left(x_{1}, \ldots, x_{n-j+1}\right) \in \mathbb{R}^{n-j+1}:\left(x_{1}, \ldots, x_{n-j}\right) \in \Gamma_{\omega^{j-1}(P)}\right\}
$$

and $\Gamma_{\omega^{i}(Q)}=\Gamma_{\omega^{i-1}(P)}$ for $j \leq i \leq n$. In particular, $W_{\omega^{i}(Q)}=W_{\omega^{i-1}(P)}$ for $j \leq i \leq n$. 
Summing up, by (3),

$$
\begin{aligned}
W_{Q}= & \bigcap_{i=0}^{n}\left[\left(\mathbb{R}^{n-i} \backslash \Gamma_{\omega^{i}(Q)}\right) \times \mathbb{R}^{i}\right] \\
= & \bigcap_{i=0}^{j-2}\left\{\left(x_{1}, \ldots, x_{n}\right) \in \mathbb{R}^{n}:\left(x_{1}, \ldots, x_{n-j}, x_{n-j+2}, \ldots, x_{n-i}\right) \in \mathbb{R}^{n-i-1} \backslash \Gamma_{\omega^{i}(P)}\right\} \\
& \quad \cap\left\{\left(x_{1}, \ldots, x_{n}\right) \in \mathbb{R}^{n}:\left(x_{1}, \ldots, x_{n-j}\right) \in \mathbb{R}^{n-j} \backslash \Gamma_{\omega^{j-1}(P)}\right\} \cap\left[W_{\omega^{j}}(Q) \times \mathbb{R}^{j}\right] \\
& \subset \bigcap_{i=0}^{j-2}\left\{\left(x_{1}, \ldots, x_{n}\right) \in \mathbb{R}^{n}:\left(x_{1}, \ldots, x_{n-j}, x_{n-j+2}, \ldots, x_{n-i}\right) \in \mathbb{R}^{n-i-1} \backslash \Gamma_{\omega^{i}(P)}\right\} \\
= & \left\{\left(x_{1}, \ldots, x_{n}\right) \in \mathbb{R}^{n}:\left(x_{i_{1}}, \ldots, x_{i_{m}}\right) \in W_{P}\right\} .
\end{aligned}
$$

This gives the assertion for $n=m+1$. Hence, by an easy induction with respect to $n-m$, we obtain the assertion.

Let $T$ be a linearly ordered set and let $\succ$ be the ordering of $T$.

For any $t_{1}, \ldots, t_{m} \in T, t_{1} \prec \cdots \prec t_{m}$, we define a projection map

$$
\pi_{t_{1}, \ldots, t_{m}}: \mathbb{R}^{T} \ni x \mapsto\left(x\left(t_{1}\right), \ldots, x\left(t_{m}\right)\right) \in \mathbb{R}^{m} .
$$

Define a family $\Omega$ of semialgebraic subsets $U$ of $\mathbb{R}^{T}$ by

$$
U=\left(\pi_{t_{1}, \ldots, t_{m}}\right)^{-1}\left(W_{P}\right),
$$

where $m \in \mathbb{N}, t_{1}, \ldots, t_{m} \in T, t_{1} \prec \cdots \prec t_{m}$, and $P \in \mathbb{Q}\left[\Lambda_{t_{1}}, \ldots, \Lambda_{t_{m}}\right] \backslash\{0\}$.

Proposition 4.3. The family $\Omega$ is a c-filter.

Proof. By Proposition 4.1 (condition $R_{2}$ ), any $U \in \Omega$ is a nonempty set.

Let $V \subsetneq \mathbb{R}^{T}$ be a $\mathbb{Q}$-algebraic set, and let $P \in \mathbb{Q}\left[\Lambda_{T}\right] \backslash\{0\}$ be such that $V=$ $\left\{x \in \mathbb{R}^{T}: P(x)=0\right\}$. Then $P \in \mathbb{Q}\left[\Lambda_{t_{1}}, \ldots, \Lambda_{t_{m}}\right] \backslash\{0\}$ for some $t_{1}, \ldots, t_{m} \in T$, $t_{1} \prec \cdots \prec t_{m}$, and $U=\left(\pi_{t_{1}, \ldots, t_{m}}\right)^{-1}\left(W_{P}\right)$. Applying Proposition 4.1 (condition $R_{0}$ ), we obtain that $U$ satisfies (i).

Let $U_{1}, U_{2} \in \Omega$. Let $t_{1}, \ldots, t_{m}, u_{1}, \ldots, u_{n} \in T$ satisfy $t_{1} \prec \cdots \prec t_{m}$ and $u_{1} \prec \cdots \prec u_{n}$, and assume moreover that for some $P \in \mathbb{Q}\left[\Lambda_{t_{1}}, \ldots, \Lambda_{t_{m}}\right]$ and $Q \in \mathbb{Q}\left[\Lambda_{u_{1}}, \ldots, \Lambda_{u_{n}}\right]$ we have $U_{1}=\left(\pi_{t_{1}, \ldots, t_{m}}\right)^{-1}\left(W_{P}\right)$ and $U_{2}=\left(\pi_{u_{1}, \ldots, u_{n}}\right)^{-1}\left(W_{Q}\right)$. Let $v_{1}, \ldots, v_{s} \in T, v_{1} \prec \cdots \prec v_{s}$, be such that $\left\{t_{1}, \ldots, t_{m}\right\} \cup\left\{u_{1}, \ldots, u_{n}\right\} \subset$ $\left\{v_{1}, \ldots, v_{s}\right\}$, and let $\bar{P}, \bar{Q} \in \mathbb{R}\left[\Lambda_{v_{1}}, \ldots, \Lambda_{v_{s}}\right]$ be polynomials of the form (4) determined by $P$ and $Q$, respectively. Then, by Proposition 4.1 (condition $R_{1}$ ) and Lemma 4.2,

$$
\left(\pi_{v_{1}, \ldots, v_{s}}\right)^{-1}\left(W_{\overline{P Q}}\right)=\left(\pi_{v_{1}, \ldots, v_{s}}\right)^{-1}\left(W_{\bar{P}}\right) \cap\left(\pi_{v_{1}, \ldots, v_{s}}\right)^{-1}\left(W_{\bar{Q}}\right) \subset U_{1} \cap U_{2} .
$$

This gives (ii). 
Take any $U \in \Omega$. There exist $t_{1}, \ldots, t_{m} \in T$ and $P \in \mathbb{R}\left[\Lambda_{t_{1}}, \ldots, \Lambda_{t_{m}}\right] \backslash\{0\}$ such that $t_{1} \prec \cdots \prec t_{m}$ and $U=\left(\pi_{t_{1}, \ldots, t_{m}}\right)^{-1}\left(W_{P}\right)$. By Proposition 4.1 (condition $R_{3}$ ), $U$ satisfies (iii). This completes the proof.

From the definition of the family $\Omega$, we immediately obtain:

Corollary 4.4. For any $t_{1}, t_{2} \in T$, we have $t_{1} \succ t_{2}$ if and only if $\Lambda_{t_{1}} \succ_{\Omega} \Lambda_{t_{2}}$.

Let $Q \in \mathbb{Q}\left[\Lambda_{T}\right] \backslash\{0\}$ and let $\Omega_{Q}$ be a family of semialgebraic subsets $U$ of $\mathbb{R}^{T}$ defined by

$$
U=\left(\pi_{t_{1}, \ldots, t_{m}}\right)^{-1}\left(W_{P} \cap W_{Q}\right),
$$

where $m \in \mathbb{N}, t_{1}, \ldots, t_{m} \in T, t_{1} \prec \cdots \prec t_{m}$, and $P Q \in \mathbb{Q}\left[\Lambda_{t_{1}}, \ldots, \Lambda_{t_{m}}\right] \backslash\{0\}$. By Proposition 4.3, we have:

Corollary 4.5. $\Omega_{Q}$ is a c-filter.

Let $X \subset \mathbb{R}^{T}$ be an open semialgebraic set and let $\stackrel{i}{x} \in X$ be a point with rational coordinates. There exist $t_{1}, \ldots, t_{k} \in T, t_{1} \prec \cdots \prec t_{k}$, and an open semialgebraic set $Y \subset \mathbb{R}^{k}$ such that $X=\left\{x \in \mathbb{R}^{T}:\left(x\left(t_{1}\right), \ldots, x\left(t_{k}\right)\right) \in Y\right\}$. Hence, there exists $r>0$ such that

$$
B:=\left\{x \in \mathbb{R}^{T}: \max _{i=1, \ldots, k}\left|x\left(t_{i}\right)-\stackrel{\circ}{x}\left(t_{i}\right)\right|<r\right\} \subset X .
$$

Let

$$
P_{0}=\Lambda_{t_{1}} \ldots \Lambda_{t_{k}}\left(\Lambda_{t_{1}}^{2}+\cdots+\Lambda_{t_{k}}^{2}-1 / r^{2}\right),
$$

let $U_{0}=\left(\pi_{t_{1}, \ldots, t_{k}}\right)^{-1}\left(W_{P_{0}}\right)$, and let $F: U_{0} \rightarrow \mathbb{R}^{T}$ be a mapping defined by

$$
F(x)(t)= \begin{cases}\stackrel{\circ}{x}(t)+1 / x(t) & \text { for } x \in U_{0}, t \in\left\{t_{1}, \ldots, t_{k}\right\}, \\ x(t) & \text { for } x \in U_{0}, t \in T \backslash\left\{t_{1}, \ldots, t_{k}\right\} .\end{cases}
$$

Proposition 4.6. $\left\{F(U): U \in \Omega_{P_{0}}\right\}$ is a c-filter subset of $X$. In particular, for any open semialgebraic set $Y \subset \mathbb{R}^{T}$, there exists $c$-filter subsets of $Y$.

Proof. By Lemma 4.2, any set $U \in \Omega_{P_{0}}$ is a subset of $U_{0}$. Moreover, $F$ is an open semialgebraic mapping, so $F(U)$ is semialgebraic for $U \in \Omega_{P_{0}}$. Hence, $\left\{F(U): U \in \Omega_{P_{0}}\right\}$ satisfies conditions (i)-(iii).

From Proposition 4.6 and Theorem 3.1, we have that:

Corollary 4.7. The set of c-filters defined in Proposition 4.6 is a dense subset of the space of orderings in $\mathbb{Q}\left(\Lambda_{T}\right)$ in the path topology of the real spectrum $\operatorname{Sper}\left(\mathbb{Q}\left[\Lambda_{T}\right]\right)$. Moreover, any ordering determined by such a c-filter is not Archimedean.

Remark 4.8. It is easy to see that the results of this section hold if we replace $\mathbb{Q}$ by $\mathbb{R}$. 


\section{Fields of Nash functions}

Let $T$ be a nonempty set. We denote by $\mathcal{N}(X)$ the domain of $\mathbb{Q}$-Nash functions on an open connected semialgebraic set $X \subset \mathbb{R}^{T}$.

Let $\succ$ be an ordering in $\mathbb{Q}\left(\Lambda_{T}\right)$ and let $\Omega_{\succ}$ be the plain filter of subsets of $\mathbb{R}^{T}$ determining $\succ$. Let us introduce in $\bigcup_{U \in \Omega_{\succ}} \mathcal{N}(U)$ a relation $\sim_{\succ}$ by

$$
\left(f_{1}: U_{1} \rightarrow \mathbb{R}\right) \sim_{\succ}\left(f_{2}: U_{2} \rightarrow \mathbb{R}\right) \Longleftrightarrow \exists_{U \in \Omega_{\succ}}\left(U \subset U_{1} \cap U_{2} \text { and }\left.f_{1}\right|_{U}=\left.f_{2}\right|_{U}\right) .
$$

From Proposition 2.4, we immediately see that $\sim_{\succ}$ is an equivalence relation. The equivalence class of $\sim_{\succ}$ determined by $f: U \rightarrow \mathbb{R}$ is denoted by $[f]_{\succ}$, and the set of all such classes by $\mathcal{N}_{\succ}$. The set $\mathcal{N}_{\succ}$ is linearly ordered by

$$
[f]_{\succ} \succ 0 \Longleftrightarrow \exists_{U \in \Omega_{\succ}}(f \in \mathcal{N}(U) \text { and } f(x)>0 \text { for } x \in U) .
$$

Proposition 5.1. The set $\mathcal{N}_{\succ}$, together with the usual operations

$$
\left[f_{1}\right]_{\succ}+\left[f_{2}\right]_{\succ}=\left[\left.f_{1}\right|_{U}+\left.f_{2}\right|_{U}\right]_{\succ}, \quad\left[f_{1}\right]_{\succ} \cdot\left[f_{2}\right]_{\succ}=\left[\left.\left.f_{1}\right|_{U} f_{2}\right|_{U}\right]_{\succ},
$$

where $f_{1} \in \mathcal{N}\left(U_{1}\right), f_{2} \in \mathcal{N}\left(U_{2}\right)$, and $U \in \Omega_{\succ}, U \subset U_{1} \cap U_{2}$, is a real field.

Proof. Since the ring $\mathcal{N}(U)$ is a domain for any $U \in \Omega_{\succ}$, so is $\mathcal{N}_{\succ}$. We prove that any nonzero $f \in \mathcal{N}_{\succ}$ has an inverse in $\mathcal{N}_{\succ}$. Indeed, there exists $U \in \Omega_{\succ}$ such that $f \in \mathcal{N}(U)$. Since $f \neq 0$, the set $f^{-1}(0)$ is contained in some proper $\mathbb{Q}$-algebraic subset of $\mathbb{R}^{T}$. Then, by the definition of c-filter, one can assume that $f(\lambda) \neq 0$ for any $\lambda \in U$. Thus $1 / f \in \mathcal{N}(U)$, so $f$ has an inverse in $\mathcal{N}_{\succ}$. Summing up, $\mathcal{N}_{\succ}$ is a field. Since $-1 \in \mathcal{N}(U)$ is not a sum of squares in $\mathcal{N}(U)$, it follows that $-1 \in \mathcal{N}_{\succ}$ is not a sum of squares in $\mathcal{N}_{\succ}$.

Theorem 5.2. The field $\mathcal{N}_{\succ}$ is a real closure of the field $\left(\mathbb{Q}\left(\Lambda_{T}\right), \succ\right)$.

Proof. Take any irreducible polynomial $P \in \mathcal{N}_{\succ}[Z]$ of odd degree $d$ with respect to $Z$. Then there exists $U \in \Omega_{\succ}$ such that $P \in \mathcal{N}(U)[Z]$. Let $t_{1}, \ldots, t_{m} \in$ $T$, and let $\tilde{U} \subset \mathbb{R}^{m}$ be an open connected semialgebraic set such that $U=$ $\left\{x \in \mathbb{R}^{T}:\left(x\left(t_{1}\right), \ldots, x\left(t_{m}\right)\right) \in \tilde{U}\right\}$. By using the Hermite method (for $\tilde{U}$ ) we deduce that there exists a decomposition $U=U_{1} \cup \cdots \cup U_{k} \cup V$ of $U$ into disjoint open basic $\mathbb{Q}$-semialgebraic sets $U_{1}, \ldots, U_{k}$ and a semialgebraic set $V$ included in an algebraic set such that $P(x, Z)$ has the same number of zeroes for all $x \in U_{i}$ and each of these zeroes is single. By (i) and (ii) in the definition of a c-filter, there exists $U^{\prime} \in \Omega_{\succ}$ such that $U^{\prime} \subset U_{i}$ for some $i \in\{1, \ldots, k\}$. Then there exists $k \in \mathbb{N}, k>0$ such that $P(x, Z)$ has exactly $k$ zeroes for $x \in U^{\prime}$, and so there exist functions $\xi_{1}, \ldots, \xi_{k}: U^{\prime} \rightarrow \mathbb{R}$ with $\xi_{1}(x)<\cdots<\xi_{k}(x)$ such that $P\left(x, \xi_{i}(x)\right)=0$ for $x \in U^{\prime}, i=1, \ldots, k$. As $\xi_{i}(x)$ are single zeroes of $P(x, Z)$, by the Implicit Function Theorem, $\xi_{i}$ is a Nash function for $i=1, \ldots, k$. As $\mathcal{N}_{\succ}$ is a real field 
(Proposition 5.1), $\mathcal{N}_{\succ}$ is a real closed field. Since $\mathcal{N}_{\succ}$ is an algebraic extension of $\mathbb{Q}\left(\Lambda_{T}\right)$, by the Artin-Schreier Theorem, it is a real closure of $\left(\mathbb{Q}\left(\Lambda_{T}\right), \succ\right)$.

Remark 5.3. The above results of this section also hold for an arbitrary c-filter determining $\succ$ in place of the plain filter $\Omega_{\succ}$. The results also hold if we put $\mathbb{R}$ in place of $\mathbb{Q}$.

From Theorems 3.1 and 5.2, we recover the familiar result that any Archimedean field can be embedded in $\mathbb{R}$.

Corollary 5.4. Let $\Omega_{\succ}$ be a plain filter of subsets of $\mathbb{R}^{T}$ determining an Archimedean ordering $\succ$ of $\mathbb{Q}\left(\Lambda_{T}\right)$, and let $x_{\succ} \in \bigcap_{U \in \Omega_{\succ}} U$. Then the mapping

$$
\mathcal{N}_{\succ} \ni f \mapsto f\left(x_{\succ}\right) \in \mathbb{R}
$$

is an order-preserving monomorphism.

From Theorem 5.2, we immediately obtain:

Corollary 5.5. Let $R$ be a real closed field with ordering $\succ$, and let $T$ be the transcendence basis of $R$ over $\mathbb{Q}$ whose existence is guaranteed by the KuratowskiZorn lemma. Assume that $T \neq \varnothing$ and let $\Lambda_{T}=\left(\Lambda_{t}: t \in T\right)$ be a system of independent variables. Then the field $R$ is order-preserving isomorphic to a real closure of the rational functions field $\mathbb{Q}\left(\Lambda_{T}\right)$, i.e., to some field $\mathcal{N}_{\succ}$.

Remark 5.6. Let $\mathbb{K}$ be an algebraically closed field of characteristic zero. Then $\mathbb{K}=R[i]$, where $i^{2}=-1$, for some real closed field $R$. Let $T \subset R$ be the transcendence basis of $\mathbb{K}$ over $\mathbb{Q}$. Assume that $T \neq \varnothing$. Then $\mathbb{K}$ is isomorphic to an algebraic closure of $\mathbb{Q}\left(\Lambda_{T}\right)$. By Theorem 1.1 of [Spodzieja 1996], one can introduce a filter $\Omega_{\mathbb{C}}$ of open, connected, and simply connected semialgebraic subsets $U$ of $\mathbb{C}^{T}$ satisfying conditions (i), (ii), and (iii). Then, analogously to [Spodzieja 1996], one can introduce a geometric construction of the algebraic closure of $\mathbb{Q}\left(\Lambda_{T}\right)$ in terms of complex Nash functions.

\section{Acknowledgements}

I would like to cordially thank Jacek Chądzyński, Michel Coste, Krzysztof Kurdyka, Tadeusz Mostowski, and Alexander Prestel for their valuable remarks and advice during the preparation of this paper.

\section{References}

[Alonso 1986] M. E. Alonso, "A note on orderings on algebraic varieties", Pacific J. Math. 123:1 (1986), 1-7. MR 87k:14020 Zbl 0562.14006

[Alonso et al. 1984] M. E. Alonso, J. M. Gamboa, and J. M. Ruiz, "Ordres sur les surfaces réelles", C. R. Acad. Sci. Paris Sér. I Math. 298:1 (1984), 17-19. MR 85b:32009 Zbl 0587.14011 
[Artin 1927] E. Artin, "Über die Zerlegung definiter Funktionen in Quadrate", Abh. Math. Sem. Univ. Hamburg 5 (1927), 100-115. JFM 52.0122.01

[Artin and Schreier 1927a] E. Artin and O. Schreier, "Algebraische Konstruktion reeller Körper", Abh. Math. Sem. Univ. Hamburg 5 (1927), 85-99. JFM 52.0120.05

[Artin and Schreier 1927b] E. Artin and O. Schreier, "Eine Kennzeichnung der reell abgeschlossenen Körper", Abh. Math. Sem. Univ. Hamburg 5 (1927), 225-331. JFM 53.0144.01

[Benedetti and Risler 1990] R. Benedetti and J.-J. Risler, Real algebraic and semi-algebraic sets, Hermann, Paris, 1990. MR 91j:14045 Zbl 0694.14006

[Bochnak and Efroymson 1980] J. Bochnak and G. A. Efroymson, "Real algebraic geometry and the 17th Hilbert problem”, Math. Ann. 251:3 (1980), 213-241. MR 81k:14023 Zbl 0425.14004

[Bochnak et al. 1987] J. Bochnak, M. Coste, and M.-F. Roy, Géométrie algébrique réelle, Ergebnisse der Mathematik und ihrer Grenzgebiete (3) 12, Springer, Berlin, 1987. MR 90b:14030 Zbl 0633.14016

[Bröker 1982] L. Bröker, "Real spectra and distributions of signatures", pp. 249-272 in Real algebraic geometry and quadratic forms (Rennes, 1981), edited by J.-L. Colliot-Thélène et al., Lecture Notes in Math. 959, Springer, Berlin, 1982. MR 84d:14014 Zbl 0501.14015

[Dubois 1981] D. W. Dubois, "Second note on Artin's solution of Hilbert's 17th problem: Order spaces”, Pacific J. Math. 97:2 (1981), 357-371. MR 83j:12017 Zbl 0476.14011

[Efroymson 1974] G. A. Efroymson, “A Nullstellensatz for Nash rings”, Pacific J. Math. 54 (1974), 101-112. MR 50 \#13024 Zbl 0321.14001

[Efroymson 1976] G. A. Efroymson, "Substitution in Nash functions", Pacific J. Math. 63:1 (1976), 137-145. MR 53 \#13211 Zbl 0335.14002

[Efroymson 1981] G. A. Efroymson, "Sums of squares in planar Nash rings", Pacific J. Math. 97:1 (1981), 75-79. MR 83b:12018 Zbl 0449.32009

[Grothendieck 1967] A. Grothendieck, "Éléments de géométrie algébrique, IV: Étude locale des schémas et des morphismes de schémas IV”, Inst. Hautes Études Sci. Publ. Math. 32 (1967), 5-361. MR 39 \#220 Zbl 0153.22301

[Guangxing 2005] Z. Guangxing, "Ordered fields satisfying Pólya's theorem”, Proc. Amer. Math. Soc. 133:10 (2005), 2921-2926. MR 2006f:12010 Zbl 1105.12002

[Marshall 2003] M. Marshall, “*-orderings and *-valuations on algebras of finite Gelfand-Kirillov dimension”, J. Pure Appl. Algebra 179:3 (2003), 255-271. MR 2004a:14062 Zbl 1052.16021

[Marshall 2008] M. Marshall, Positive polynomials and sums of squares, Mathematical Surveys and Monographs 146, Amer. Math. Soc., Providence, RI, 2008. MR 2009a:13044 Zbl 1169.13001

[Mostowski 1976] T. Mostowski, "Some properties of the ring of Nash functions", Ann. Scuola Norm. Sup. Pisa Cl. Sci. (4) 3:2 (1976), 245-266. MR 54 \#307 Zbl 0335.14001

[Nash 1952] J. Nash, "Real algebraic manifolds", Ann. of Math. (2) 56 (1952), 405-421. MR 14,403b Zbl 0048.38501

[Prestel and Delzell 2001] A. Prestel and C. N. Delzell, Positive polynomials: from Hilbert's 17th problem to real algebra, Springer, Berlin, 2001. MR 2002k:13044 Zbl 0987.13016

[Schwartz 1980] N. Schwartz, "Archimedean lattice-ordered fields that are algebraic over their o-subfields”, Pacific J. Math. 89:1 (1980), 189-198. MR 82e:06018 Zbl 0442.06008

[Seidenberg 1954] A. Seidenberg, "A new decision method for elementary algebra", Ann. of Math. (2) 60 (1954), 365-374. MR 16,209a Zbl 0056.01804

[Spodzieja 1996] S. Spodzieja, "The field of Nash functions and factorization of polynomials", Ann. Polon. Math. 65:1 (1996), 81-94. MR 97h:12006 Zbl 0909.12002 
[Tancredi and Tognoli 2006] A. Tancredi and A. Tognoli, "On the products of Nash subvarieties by spheres", Proc. Amer. Math. Soc. 134:4 (2006), 983-987. MR 2006i:14062 Zbl 1093.14079

[Tarski 1948] A. Tarski, A decision method for elementary algebra and geometry, RAND, Santa Monica, CA, 1948. MR 10,499f Zbl 0035.00602

[Tworzewski 1990] P. Tworzewski, "Intersections of analytic sets with linear subspaces", Ann. Scuola Norm. Sup. Pisa Cl. Sci. (4) 17:2 (1990), 227-271. MR 91j:32008 Zbl 0717.32006

Received June 11, 2012. Revised September 25, 2012.

STANISŁAW SPODZIEJA

FACULTY OF MATHEMATICS AND COMPUTER SCIENCE

DEPARTMENT OF ANALYTIC FUnCTIONS AND DifFERENTIAL EQUATIONS

UNIVERSITY OF ŁÓDŹ

S. BANACHA 22

90-238 ŁóDŹ

POLAND

spodziej@math.uni.lodz.pl 


\title{
PACIFIC JOURNAL OF MATHEMATICS
}

\author{
msp.org/pjm
}

Founded in 1951 by E. F. Beckenbach (1906-1982) and F. Wolf (1904-1989)

\section{EDITORS}

V. S. Varadarajan (Managing Editor)

Department of Mathematics

University of California

Los Angeles, CA 90095-1555

pacific@math.ucla.edu

Paul Balmer

Department of Mathematics

University of California

Los Angeles, CA 90095-1555

balmer@math.ucla.edu

Daryl Cooper

Department of Mathematics

University of California

Santa Barbara, CA 93106-3080 cooper@math.ucsb.edu

Jiang-Hua $\mathrm{Lu}$

Department of Mathematics

The University of Hong Kong

Pokfulam Rd., Hong Kong jhlu@maths.hku.hk
Don Blasius

Department of Mathematics University of California

Los Angeles, CA 90095-1555

blasius@math.ucla.edu

Robert Finn

Department of Mathematics Stanford University

Stanford, CA 94305-2125

finn@math.stanford.edu

Sorin Popa

Department of Mathematics

University of California

Los Angeles, CA 90095-1555

popa@math.ucla.edu

Paul Yang

Department of Mathematics

Princeton University

Princeton NJ 08544-1000

yang@math.princeton.edu

\section{PRODUCTION}

Silvio Levy, Scientific Editor, production@msp.org

\section{SUPPORTING INSTITUTIONS}

ACADEMIA SINICA, TAIPEI

CALIFORNIA INST. OF TECHNOLOGY

INST. DE MATEMÁTICA PURA E APLICADA

KEIO UNIVERSITY

MATH. SCIENCES RESEARCH INSTITUTE

NEW MEXICO STATE UNIV.

OREGON STATE UNIV.

\author{
STANFORD UNIVERSITY \\ UNIV. OF BRITISH COLUMBIA \\ UNIV. OF CALIFORNIA, BERKELEY \\ UNIV. OF CALIFORNIA, DAVIS \\ UNIV. OF CALIFORNIA, LOS ANGELES \\ UNIV. OF CALIFORNIA, RIVERSIDE \\ UNIV. OF CALIFORNIA, SAN DIEGO \\ UNIV. OF CALIF., SANTA BARBARA
}

\author{
Vyjayanthi Chari \\ Department of Mathematics \\ University of California \\ Riverside, CA 92521-0135 \\ chari@math.ucr.edu \\ Kefeng Liu \\ Department of Mathematics \\ University of California \\ Los Angeles, CA 90095-1555 \\ liu@math.ucla.edu \\ Jie Qing \\ Department of Mathematics \\ University of California \\ Santa Cruz, CA 95064 \\ qing@cats.ucsc.edu
}

These supporting institutions contribute to the cost of publication of this Journal, but they are not owners or publishers and have no responsibility for its contents or policies.

See inside back cover or msp.org/pjm for submission instructions.

The subscription price for 2013 is US \$400/year for the electronic version, and \$485/year for print and electronic.

Subscriptions, requests for back issues and changes of subscribers address should be sent to Pacific Journal of Mathematics, P.O. Box 4163, Berkeley, CA 94704-0163, U.S.A. The Pacific Journal of Mathematics is indexed by Mathematical Reviews, Zentralblatt MATH, PASCAL CNRS Index, Referativnyi Zhurnal, Current Mathematical Publications and the Science Citation Index.

The Pacific Journal of Mathematics (ISSN 0030-8730) at the University of California, c/o Department of Mathematics, 798 Evans Hall \#3840, Berkeley, CA 94720-3840, is published ten to twelve times a year. Periodical rate postage paid at Berkeley, CA 94704, and additional mailing offices. POSTMASTER: send address changes to Pacific Journal of Mathematics, P.O. Box 4163, Berkeley, CA 94704-0163.

PJM peer review and production are managed by EditFLOW ${ }^{\circledR}$ from Mathematical Sciences Publishers.

PUBLISHED BY 


\section{PACIFIC JOURNAL OF MATHEMATICS}

Volume $264 \quad$ No. $2 \quad$ August 2013

On 4-manifolds, folds and cusps

STEFAN BEHRENS

Thin $r$-neighborhoods of embedded geodesics with finite length and

307 negative Jacobi operator are strongly convex

PHILIPPE DELANOË

Eigenvalues of perturbed Laplace operators on compact manifolds

ASMA HASSANNEZHAD

Four equivalent versions of nonabelian gerbes

THOMAS NIKOLAUS and KONRAD WALDORF

On nonlinear nonhomogeneous resonant Dirichlet equations

421

Nikolaos S. PAPAGEORGIOU and GEORGE SMYRLIS

A geometric model of an arbitrary real closed field

455

STANISŁAW SPODZIEJA

Twisted K-theory for the orbifold $[* / G]$

471

MARIO VELÁSQUEZ, EdWARd BECERRA and HERMES

MARTINEZ

Linear restriction estimates for the wave equation with an inverse square potential

JUNYONG ZHANG and JIQIANG ZHENG 